\title{
Some fractional extensions of trapezium inequalities via coordinated harmonic convex functions
}

\author{
Muhammad Uzair Awan ${ }^{a, *}$, Muhammad Aslam Noor ${ }^{b, c}$, Marcela V. Mihaid ${ }^{d}$, Khalida Inayat Noor \\ ${ }^{a}$ Government College University, Faisalabad, Pakistan. \\ ${ }^{b}$ Mathematics Department, King Saud University, Riyadh, Saudi Arabia. \\ ${ }^{c}$ Mathematics Department, COMSATS Institute of Information, Technology, Islamabad, Pakistan. \\ ${ }^{d}$ Department scientific-methodical sessions, Romanian Mathematical Society-branch Bucharest, Academy Street no. 14, RO-010014, \\ Bucharest, Romania.

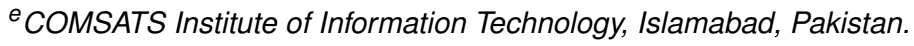

Communicated by $\mathrm{Sh}$. Wu

\begin{abstract}
In this article, we derive a new fractional estimate for Hermite-Hadamard's inequality via coordinated harmonic convex functions on a rectangle from the plane $\mathbb{R}^{2}$. We establish a new fractional integral identity for partially differentiable functions. Utilizing this integral identity, we obtain some more fractional estimates for Hermite-Hadamard's inequality. The ideas of this paper may stimulate further research. (C)2017 All rights reserved.
\end{abstract}

Keywords: Harmonic convex functions, fractional, trapezium, Hermite-Hadamard inequalities. 2010 MSC: 26D15, 26A51, 26A33.

\section{Introduction and preliminaries}

Let $f: I=[a, b] \subset \mathbb{R} \rightarrow \mathbb{R}$ be a convex function, then

$$
f\left(\frac{a+b}{2}\right) \leqslant \frac{1}{b-a} \int_{a}^{b} f(x) d x \leqslant \frac{f(a)+f(b)}{2} .
$$

This above result is due to Hermite and Hadamard independently and is well-known as Hermite-Hadamard's inequality. This inequality is also known as trapezium inequality. It provides an equivalent property for convexity. Due to its rich applications, especially in the field of numerical analysis, this result has attracted many researchers from all over the world. In recent decades, we have seen a rapid development in inequality theory. Many inequalities can be obtained for convex functions. However

\footnotetext{
*Corresponding author

Email addresses: awan.uzair@gmail.com (Muhammad Uzair Awan), noormaslam@gmail.com (Muhammad Aslam Noor), marcelamihai58@yahoo.com (Marcela V. Mihai), khalidanoor@hotmail.com (Khalida Inayat Noor)

doi:10.22436/jnsa.010.04.34
} 
among those, Hermite-Hadamard's inequality is one of the most extensively and intensively studied result. The point of pondering is that there is a close relationship between theory of inequalities and theory of convexity. This fact makes the theory of inequalities more fascinating and interesting. There are several new extensions and generalizations of classical convexity considered in recent years and one can observe that parallel to this development in theory of convexity, theory of inequalities particularly integral inequalities theory developed rapidly, for example see [1-6, 8, 10, 11, 13-23]. Iscan [6] introduced the notion of harmonic convex functions which opened a new venue for researchers. Noor and Noor [12] proved that the optimality conditions of the differentiable harmonic convex functions can be characterized by a class of variational inequalities, which is called harmonic variational inequalities. Iscan [6] derived a new extension for Hermite-Hadamard's inequality. Noor et al. [14] extended the class of harmonic convex function on coordinates and introduced the class of coordinated harmonic convex functions. They have also obtained some new estimates for Hermite-Hadamard's inequality essentially using the class of coordinated harmonic convex function. Sarikaya et al. [21] gave some new refinements for classical Hermite-Hadamard's inequality using Riemann-Liouville integrals. Utilizing the idea of Sarikaya [19], Iscan and $\mathrm{Wu}$ [7] derived the fractional estimate of Hermite-Hadamard's inequality for harmonic convex functions. Also Sarikaya [19] obtained the fractional estimate for Hermite-Hadamard's inequality for coordinated convex functions. The main motivation of this article is to give some new fractional estimates for Hermite-Hadamard's inequality via harmonic convex functions. It is hoped that the ideas used in this paper may be helpful for further research in this field.

Before proceeding to our main results, we recall some preliminary concepts and results. These will be helpful in obtaining our main results. First of all, we define harmonic convex functions.

Definition 1.1 ([6]). A function $f: K_{h} \subset \mathbb{R}_{+} \rightarrow \mathbb{R}$ is said to be harmonic convex function, if

$$
f\left(\frac{x y}{t x+(1-t) y}\right) \leqslant(1-t) f(x)+t f(y), \quad \forall x, y \in K_{h}, \quad t \in[0,1] .
$$

The following inequality is Hermite-Hadamard's inequality for harmonic convex functions.

Theorem 1.2 ([6]). Let $f: \mathcal{J}_{h}=[a, b] \subset \mathbb{R}_{+} \rightarrow \mathbb{R}$ be an integrable harmonic convex function, then

$$
f\left(\frac{2 a b}{a+b}\right) \leqslant \frac{a b}{b-a} \int_{a}^{b} \frac{f(x)}{x^{2}} d x \leqslant \frac{f(a)+f(b)}{2} .
$$

Noor et al. [14] has given the following definition for coordinated harmonic convex functions.

Definition 1.3. Consider the rectangle $\Delta=[a, b] \times[c, d] \subset \mathbb{R}^{2}$. A function $f: \Delta \rightarrow \mathbb{R}$ is said to be coordinated harmonically convex function on $\Delta$, if

$$
f\left(\frac{x y}{t x+(1-t) y}, \frac{u w}{r u+(1-r) w}\right) \leqslant(1-t)(1-r) f(x, u)+r(1-t) f(x, w)+t(1-r) f(y, u)+\operatorname{trf}(y, w),
$$

whenever $x, y \in[a, b], u, w \in[c, d]$ and $t, r \in[0,1]$.

We now recall some results and concepts from fractional calculus. The classical form of fractional calculus is given by Riemann-Liouville integrals, which are defined as:

Definition 1.4 ([9]). Let $f \in L_{1}[a, b]$. Then the Riemann-Liouville integrals $J_{a^{+}}^{\alpha} f$ and $J_{b^{-}}^{\alpha} f$ of order $\alpha>0$ with $a \geqslant 0$ are defined by

$$
J_{a^{+}}^{\alpha} f(x)=\frac{1}{\Gamma(\alpha)} \int_{a}^{x}(x-t)^{\alpha-1} f(t) d t, \quad x>a,
$$

and

$$
J_{b^{-}}^{\alpha} f(x)=\frac{1}{\Gamma(\alpha)} \int_{x}^{b}(t-x)^{\alpha-1} f(t) d t, \quad x<b,
$$

where 


$$
\Gamma(\alpha)=\int_{0}^{\infty} e^{-t} x^{\alpha-1} \mathrm{~d} x
$$

is the well-known Gamma function.

Definition 1.5 ([19]). Let $f \in \mathrm{L}_{1}([a, b],[c, d])$. The Riemann-Liouville integrals $\mathrm{J}_{\mathrm{a}^{+}, \mathrm{c}^{+},}^{\alpha, \beta} \mathrm{J}_{\mathrm{a}^{+}, \mathrm{d}^{-},}^{\alpha, \beta} \mathrm{J}_{\mathrm{b}^{-}, \mathrm{c}^{+}}^{\alpha, \beta}$ and $\mathrm{J}_{\mathrm{b}^{-}, \mathrm{d}^{-}}^{\alpha, \beta}$ of order $\alpha, \beta>0$ with $\mathrm{a}, \mathrm{c} \geqslant 0$ are defined by

$$
\begin{aligned}
& J_{a^{+}, c^{+}}^{\alpha, \beta} f(x, y)=\frac{1}{\Gamma(\alpha) \Gamma(\beta)} \int_{a}^{x} \int_{c}^{y}(x-t)^{\alpha-1}(y-s)^{\beta-1} f(t, s) d s d t, \quad x>a, y>c, \\
& J_{a^{+}, d^{-}}^{\alpha, \beta} f(x, y)=\frac{1}{\Gamma(\alpha) \Gamma(\beta)} \int_{a}^{x} \int_{y}^{d}(x-t)^{\alpha-1}(s-y)^{\beta-1} f(t, s) d s d t, \quad x>a, y<d, \\
& \mathrm{~J}_{\mathrm{b}^{-}, \mathrm{c}^{+}}^{\alpha, \beta} \mathrm{f}(\mathrm{x}, \mathrm{y})=\frac{1}{\Gamma(\alpha) \Gamma(\beta)} \int_{x}^{\mathrm{b}} \int_{\mathrm{c}}^{\mathrm{y}}(\mathrm{t}-\mathrm{x})^{\alpha-1}(y-s)^{\beta-1} \mathrm{f}(\mathrm{t}, \mathrm{s}) \mathrm{dsdt}, \quad x<\mathrm{b}, \quad y>\mathrm{c}, \\
& \mathrm{J}_{\mathrm{b}^{-}, \mathrm{d}^{-}}^{\alpha, \beta} \mathrm{f}(\mathrm{x}, \mathrm{y})=\frac{1}{\Gamma(\alpha) \Gamma(\beta)} \int_{x}^{\mathrm{b}} \int_{y}^{\mathrm{d}}(\mathrm{t}-x)^{\alpha-1}(s-y)^{\beta-1} f(t, s) \mathrm{dsdt}, \quad x<\mathrm{b}, \quad y<d,
\end{aligned}
$$

respectively.

Also,

$$
\begin{aligned}
& J_{a^{+}}^{\alpha} f\left(x, \frac{c+d}{2}\right)=\frac{1}{\Gamma(\alpha)} \int_{a}^{x}(x-t)^{\alpha-1} f\left(t, \frac{c+d}{2}\right) d t, \quad x>a \\
& J_{b^{-}}^{\alpha} f\left(x, \frac{c+d}{2}\right)=\frac{1}{\Gamma(\alpha)} \int_{x}^{b}(t-x)^{\alpha-1} f\left(t, \frac{c+d}{2}\right) d t, \quad x<b \\
& J_{c^{+}}^{\alpha} f\left(\frac{a+b}{2}, y\right)=\frac{1}{\Gamma(\beta)} \int_{c}^{y}(y-s)^{\beta-1} f\left(\frac{a+b}{2}, s\right) d s, \quad y>c \\
& J_{d^{-}}^{\alpha} f\left(\frac{a+b}{2}, y\right)=\frac{1}{\Gamma(\beta)} \int_{y}^{d}(s-y)^{\beta-1} f\left(\frac{a+b}{2}, s\right) d s, \quad y<d .
\end{aligned}
$$

For the reader's convenience, we recall the definition of the Beta function $\mathrm{B}(.,$.

$$
B(x, y)=\int_{0}^{1} t^{x-1}(1-t)^{y-1} d t \frac{\Gamma(x) \Gamma(y)}{\Gamma(x+y)} .
$$

The integral form of the hypergeometric function is

$$
{ }_{2} F_{1}(x, y ; c ; z)=\frac{1}{B(y, c-y)} \int_{0}^{1} t^{y-1}(1-t)^{c-y-1}(1-z t)^{-x} d t
$$

for $|z|<1, c>y>0$.

Lemma 1.6 ([6]). For $0<\alpha \leqslant 1$ and $0 \leqslant a<b$, we have

$$
\left|a^{\alpha}-b^{\alpha}\right| \leqslant(b-a)^{\alpha} .
$$

\section{A new two dimensional fractional integral identity}

In this section, we derive a new fractional integral identity. 
Lemma 2.1. Let $\mathrm{f}: \Delta \subset(0, \infty) \times(0, \infty) \rightarrow \mathbb{R}$ be partial differentiable function on $\Delta$ with $\mathrm{a}<\mathrm{b}$ and $\mathrm{c}<\mathrm{d}$. If $\frac{\partial^{2} \mathrm{f}}{\partial \mathrm{t} \partial \mathrm{r}} \in \mathscr{L}(\Delta)$, then the following fractional integral identity holds:

$$
\begin{aligned}
\Xi_{f}(\Delta ; \Gamma ; \alpha, \beta)= & \frac{a b(b-a) c d(d-c)}{4} \\
& \times\left\{\int_{0}^{1} \int_{0}^{1}\left(\frac{t^{\alpha}-(1-t)^{\alpha}}{A_{t}^{2}}\right)\left(\frac{r^{\beta}-(1-r)^{\beta}}{A_{r}^{2}}\right) \frac{\partial^{2} f}{\partial t \partial r}\left(\frac{a b}{A_{t}}, \frac{c d}{A_{r}}\right) d r d t\right\},
\end{aligned}
$$

where

$$
\begin{aligned}
\Xi_{f}(\Delta ; \Gamma ; \alpha, \beta)= & \frac{f(a, c)+f(a, d)+f(b, c)+f(b, d)}{4} \\
& -\frac{\Gamma(\beta+1)(c d)^{\beta}}{4(d-c)^{\beta}}\left[J_{\frac{1}{d^{+}}}^{\beta} f\left(b, \frac{1}{c}\right)+J_{\frac{1}{c^{-}}}^{\beta} f\left(b, \frac{1}{d}\right)+J_{\frac{1}{d^{+}}}^{\beta} f\left(a, \frac{1}{c}\right)+J_{\frac{1}{c^{-}}}^{\beta} f\left(a, \frac{1}{d}\right)\right] \\
& -\frac{\Gamma(\alpha+1)(a b)^{\alpha}}{4(b-a)^{\alpha}}\left[J_{\frac{1}{b^{+}}}^{\alpha} f\left(\frac{1}{a}, d\right)+J_{\frac{1}{b^{+}}}^{\alpha} f\left(\frac{1}{a}, c\right)+J_{\frac{1}{a^{-}}}^{\alpha} f\left(\frac{1}{b}, d\right)+J_{\frac{1}{a^{-}}}^{\alpha} f\left(\frac{1}{b}, c\right)\right] \\
& +\frac{(a b)^{\alpha} \Gamma(\alpha+1)(c d)^{\beta} \Gamma(\beta+1)}{4(b-a)^{\alpha}(d-c)^{\beta}}\left[J_{\frac{1}{b^{+}}, \frac{1}{d^{+}}}^{\alpha, \beta} f\left(\frac{1}{a}, \frac{1}{c}\right)+J_{\frac{1}{b^{+}}, \frac{1}{c^{-}}}^{\alpha, \beta} f\left(\frac{1}{a}, \frac{1}{d}\right)\right. \\
& \left.+J_{\frac{1}{a^{-}}, \frac{1}{d^{+}}}^{\alpha, \beta} f\left(\frac{1}{b}, \frac{1}{c}\right)+J_{\frac{1}{a^{-}}, \frac{1}{c^{-}}}^{\alpha, \beta} f\left(\frac{1}{b}, \frac{1}{d}\right)\right] .
\end{aligned}
$$

Proof. Let

$$
\begin{aligned}
K:= & \frac{a b(b-a) c d(d-c)}{4} \\
& \times\left\{\int_{0}^{1} \int_{0}^{1}\left(\frac{t^{\alpha}-(1-t)^{\alpha}}{A_{t}^{2}}\right)\left(\frac{r^{\beta}-(1-r)^{\beta}}{A_{r}^{2}}\right) \frac{\partial^{2} f}{\partial t \partial r}\left(\frac{a b}{A_{t}}, \frac{c d}{A_{r}}\right) d r d t\right\} \\
= & \frac{a b(b-a) c d(d-c)}{4} \iint_{0}^{1}\left\{\frac{t^{\alpha} r^{\beta}}{A_{t}^{2} A_{r}^{2}}-\frac{t^{\alpha}(1-r)^{\beta}}{A_{t}^{2} A_{r}^{2}}-\frac{(1-t)^{\alpha} r^{\beta}}{A_{t}^{2} A_{r}^{2}}+\frac{(1-t)^{\alpha}(1-r)^{\beta}}{A_{t}^{2} A_{r}^{2}}\right\} \\
& \times \frac{\partial^{2} f}{\partial t \partial r}\left(\frac{a b}{A_{t}}, \frac{c d}{A_{r}}\right) d r d t \\
= & \frac{a b(b-a) c d(d-c)}{4}\left(K_{1}-K_{2}-K_{3}+K_{4}\right),
\end{aligned}
$$

where

$$
\begin{aligned}
K_{1}:= & \int_{0}^{1} \int_{0}^{1} \frac{t^{\alpha} r^{\beta}}{A_{t}^{2} A_{r}^{2}} \frac{\partial^{2} f}{\partial t \partial r}\left(\frac{a b}{A_{t}}, \frac{c d}{A_{r}}\right) d r d t \\
= & \frac{f(b, d)}{a b(b-a) c d(d-c)}-\frac{\beta}{a b(b-a) c d(d-c)} \int_{0}^{1} r^{\beta-1} f\left(b, \frac{c d}{A_{r}}\right) d r \\
& -\frac{\alpha}{a b(b-a) c d(d-c)} \int_{0}^{1} t^{\alpha-1} f\left(\frac{a b}{A_{t}}, d\right) d t \\
& +\frac{\alpha \beta}{a b(b-a) c d(d-c)} \int_{0}^{1} \int_{0}^{1} t^{\alpha-1} r^{\beta-1} f\left(\frac{a b}{A_{t}}, \frac{c d}{A_{r}}\right) d r d t
\end{aligned}
$$




$$
\begin{aligned}
& K_{2}:=\int_{0}^{1} \int_{0}^{1} \frac{t^{\alpha}(1-r)^{\beta}}{A_{t}^{2} A_{r}^{2}} \frac{\partial^{2} f}{\partial t \partial r}\left(\frac{a b}{A_{t}}, \frac{c d}{A_{r}}\right) d r d t \\
& =-\frac{f(b, c)}{a b(b-a) c d(d-c)}-\frac{\beta}{a b(b-a) c d(d-c)} \int_{0}^{1}(1-r)^{\beta-1} f\left(b, \frac{c d}{A_{r}}\right) d r \\
& +\frac{\alpha}{a b(b-a) c d(d-c)} \int_{0}^{1} t^{\alpha-1} f\left(\frac{a b}{A_{t}}, c\right) d t \\
& +\frac{\alpha \beta}{a b(b-a) c d(d-c)} \int_{0}^{1} \int_{0}^{1} t^{\alpha-1}(1-r)^{\beta-1} f\left(\frac{a b}{A_{t}}, \frac{c d}{A_{r}}\right) d r d t \\
& K_{3}:=\int_{0}^{1} \int_{0}^{1} \frac{(1-t)^{\alpha} r^{\beta}}{A_{t}^{2} A_{r}^{2}} \frac{\partial^{2} f}{\partial t \partial r}\left(\frac{a b}{A_{t}}, \frac{c d}{A_{r}}\right) d r d t \\
& =-\frac{f(a, d)}{a b(b-a) c d(d-c)}+\frac{\beta}{a b(b-a) c d(d-c)} \int_{0}^{1} r^{\beta-1} f\left(a, \frac{c d}{A_{r}}\right) d r \\
& -\frac{\alpha}{a b(b-a) c d(d-c)} \int_{0}^{1}(1-t)^{\alpha-1} f\left(\frac{a b}{A_{t}}, d\right) d t \\
& +\frac{\alpha \beta}{a b(b-a) c d(d-c)} \int_{0}^{1} \int_{0}^{1}(1-t)^{\alpha-1} r^{\beta-1} f\left(\frac{a b}{A_{t}}, \frac{c d}{A_{r}}\right) d r d t,
\end{aligned}
$$

and

$$
\begin{aligned}
\mathrm{K}_{4}: & =\int_{0}^{1} \int_{0}^{1} \frac{(1-t)^{\alpha}(1-r)^{\beta}}{A_{t}^{2} A_{r}^{2}} \frac{\partial^{2} f}{\partial t \partial r}\left(\frac{a b}{A_{t}}, \frac{c d}{A_{r}}\right) d r d t \\
= & \frac{f(a, c)}{a b(b-a) c d(d-c)}+\frac{\beta}{a b(b-a) c d(d-c)} \int_{0}^{1}(1-r)^{\beta-1} f\left(a, \frac{c d}{A_{r}}\right) d r \\
& -\frac{\alpha}{a b(b-a) c d(d-c)} \int_{0}^{1}(1-t)^{\alpha-1} f\left(\frac{a b}{A_{t}}, c\right) d t \\
& +\frac{\alpha \beta}{a b(b-a) c d(d-c)} \int_{0}^{1} \int_{0}^{1}(1-t)^{\alpha-1}(1-r)^{\beta-1} f\left(\frac{a b}{A_{t}}, \frac{c d}{A_{r}}\right) d r d t .
\end{aligned}
$$

Now changing the variables and using (2.2), (2.3), (2.4) and (2.5) in (2.1), we get

$$
\begin{aligned}
K:= & \frac{f(a, c)+f(a, d)+f(b, c)+f(b, d)}{4} \\
& -\frac{\Gamma(\beta+1)(c d)^{\beta}}{4(d-c)^{\beta}}\left[J_{\frac{1}{d^{+}}}^{\beta} f\left(b, \frac{1}{c}\right)+J_{\frac{1}{c^{-}}}^{\beta} f\left(b, \frac{1}{d}\right)+J_{\frac{1}{d^{+}}}^{\beta} f\left(a, \frac{1}{c}\right)+J_{\frac{1}{c^{-}}}^{\beta} f\left(a, \frac{1}{d}\right)\right] \\
& -\frac{\Gamma(\alpha+1)(a b)^{\alpha}}{4(b-a)^{\alpha}}\left[J_{\frac{1}{b^{+}}}^{\alpha} f\left(\frac{1}{a}, d\right)+J_{\frac{1}{b^{+}}}^{\alpha} f\left(\frac{1}{a^{2}}, c\right)+J_{\frac{1}{a^{-}}}^{\alpha} f\left(\frac{1}{b}, d\right)+J_{\frac{1}{a^{-}}}^{\alpha} f\left(\frac{1}{b}, c\right)\right]
\end{aligned}
$$




$$
\begin{aligned}
& +\frac{(a b)^{\alpha} \Gamma(\alpha+1)(c d)^{\beta} \Gamma(\beta+1)}{4(b-a)^{\alpha}(d-c)^{\beta}}\left[J_{\frac{1}{b^{+}, \frac{1}{d^{+}}}}^{\alpha, \beta} f\left(\frac{1}{a}, \frac{1}{c}\right)+J_{\frac{1}{b^{+}}, \frac{1}{c^{-}}}^{\alpha, \beta} f\left(\frac{1}{a}, \frac{1}{d}\right)\right. \\
& \left.+J_{\frac{1}{a^{-}, \frac{1}{d^{+}}}}^{\alpha, \beta} f\left(\frac{1}{b}, \frac{1}{c}\right)+J_{\frac{1}{a^{-}}, \frac{1}{c^{-}}}^{\alpha, \beta} f\left(\frac{1}{b}, \frac{1}{d}\right)\right] .
\end{aligned}
$$

This completes the proof.

Note that if $\alpha=1=\beta$, then Lemma 2.1 reduces to integral identity which involves ordinary integrals, for more details see [14].

\section{Main results}

In this section, we present our new extensions of Hermite-Hadamard type inequalities via coordinated harmonic convex functions. These results involve two dimensional Riemann-Liouville fractional integrals.

Theorem 3.1. Let $\mathrm{f}: \Delta \subset(0, \infty) \times(0, \infty) \rightarrow \mathbb{R}$ be coordinated harmonic convex function. If $\mathrm{f} \in \mathscr{L}(\Delta)$, then the following fractional estimates of Hermite-Hadamard inequality holds:

$$
\begin{aligned}
f\left(\frac{2 a b}{a+b}, \frac{2 c d}{c+d}\right) \leqslant & \frac{\Gamma(\alpha+1) \Gamma(\beta+1)}{4}\left(\frac{a b}{b-a}\right)^{\alpha}\left(\frac{c d}{d-c}\right)^{\beta}\left[J_{\frac{1}{b^{+}, \frac{1}{d^{+}}}}^{\alpha, \beta}(f \circ g)\left(\frac{1}{a^{\prime}}, \frac{1}{c}\right)\right. \\
& \left.+J_{\frac{1}{b^{+}, \frac{1}{c^{-}}}}^{\alpha, \beta}(f \circ g)\left(\frac{1}{a^{\prime}}, \frac{1}{d}\right)+J_{\frac{1}{a^{-}, \frac{1}{d^{+}}}}^{\alpha, \beta}(f \circ g)\left(\frac{1}{b^{\prime}}, \frac{1}{c}\right)+J_{\frac{1}{a^{-}, \frac{1}{c^{-}}}}^{\alpha, \beta}(f \circ g)\left(\frac{1}{b^{\prime}}, \frac{1}{d}\right)\right] \\
\leqslant & \frac{f(a, c)+f(a, d)+f(b, c)+f(b, d)}{4} .
\end{aligned}
$$

Proof. Since $\mathrm{f}$ is a coordinated harmonically convex function, then, using $t=r=\frac{1}{2}$ and $x=\frac{a b}{t_{1} a+\left(1-t_{1}\right) b}$, $y=\frac{a b}{\left(1-t_{1}\right) a+t_{1} b}, u=\frac{c d}{r_{1} c+\left(1-r_{1}\right) d}$ and $w=\frac{c d}{\left(1-r_{1}\right) a+r_{1} d}$, we have

$$
\begin{aligned}
f\left(\frac{2 a b}{a+b}, \frac{2 c d}{c+d}\right) \leqslant & \frac{1}{4}\left[f\left(\frac{a b}{t_{1} a+\left(1-t_{1}\right) b}, \frac{c d}{r_{1} c+\left(1-r_{1}\right) d}\right)+f\left(\frac{a b}{t_{1} a+\left(1-t_{1}\right) b}, \frac{c d}{\left(1-r_{1}\right) c+r_{1} d}\right)\right. \\
& \left.+f\left(\frac{a b}{\left(1-t_{1}\right) a+t_{1} b^{\prime}}, \frac{c d}{r_{1} c+\left(1-r_{1}\right) d}\right)+f\left(\frac{a b}{\left(1-t_{1}\right) a+t_{1} b}, \frac{c d}{\left(1-r_{1}\right) c+r_{1} d}\right)\right] .
\end{aligned}
$$

Multiplying both sides of inequality (3.1) with $t_{1}^{\alpha-1} r_{1}^{\beta-1}$ and then integrating with respect to $\left(t_{1}, r_{1}\right)$ on $[0,1] \times[0,1]$, we have

$$
\begin{aligned}
\frac{1}{\alpha \beta} f\left(\frac{2 a b}{a+b}, \frac{2 c d}{c+d}\right) \leqslant & \frac{1}{4} \int_{0}^{1} \int_{0}^{1} t_{1}^{\alpha-1} r_{1}^{\beta-1}\left[f\left(\frac{a b}{t_{1} a+\left(1-t_{1}\right) b}, \frac{c d}{r_{1} c+\left(1-r_{1}\right) d}\right)\right. \\
& +f\left(\frac{a b}{t_{1} a+\left(1-t_{1}\right) b}, \frac{c d}{\left(1-r_{1}\right) c+r_{1} d}\right)+f\left(\frac{a b}{\left(1-t_{1}\right) a+t_{1} b}, \frac{c d}{r_{1} c+\left(1-r_{1}\right) d}\right) \\
& \left.+f\left(\frac{a b}{\left(1-t_{1}\right) a+t_{1} b}, \frac{c d}{\left(1-r_{1}\right) c+r_{1} d}\right)\right] d r_{1} d t_{1} .
\end{aligned}
$$

This implies

$$
\begin{aligned}
f\left(\frac{2 a b}{a+b}, \frac{2 c d}{c+d}\right) \leqslant & \frac{\Gamma(\alpha+1) \Gamma(\beta+1)}{4}\left(\frac{a b}{b-a}\right)^{\alpha}\left(\frac{c d}{d-c}\right)^{\beta}\left[J_{\frac{1}{b^{+}, \frac{1}{d^{+}}}}^{\alpha, \beta}(f \circ g)\left(\frac{1}{a}, \frac{1}{c}\right)\right. \\
& \left.+J_{\frac{1}{b^{+}, \frac{1}{c^{-}}}}^{\alpha, \beta}(f \circ g)\left(\frac{1}{a}, \frac{1}{d}\right)+J_{\frac{1}{a^{-}, \frac{1}{d^{+}}}}^{\alpha, \beta}(f \circ g)\left(\frac{1}{b^{\prime}}, \frac{1}{c}\right)+J_{\frac{1}{a^{-}, \frac{1}{c^{-}}}}^{\alpha, \beta}(f \circ g)\left(\frac{1}{b^{\prime}}, \frac{1}{d}\right)\right] .
\end{aligned}
$$


Also

$$
\begin{aligned}
& f\left(\frac{a b}{t a+(1-t) b}, \frac{c d}{r c+(1-r) d}\right)+f\left(\frac{a b}{t a+(1-t) b}, \frac{c d}{(1-r) c+r d}\right) \\
&+f\left(\frac{a b}{(1-t) a+t b}, \frac{c d}{r c+(1-r) d}\right)+f\left(\frac{a b}{(1-t) a+t b}, \frac{c d}{(1-r) c+r d}\right) \\
& \leqslant f(a, c)+f(a, d)+f(b, c)+f(b, d) .
\end{aligned}
$$

Multiplying both sides of above inequality with $t^{\alpha-1} r^{\beta-1}$ and then integrating with respect to $(t, r)$ on $[0,1] \times[0,1]$, we have

$$
\begin{gathered}
\frac{\Gamma(\alpha+1) \Gamma(\beta+1)}{4}\left(\frac{a b}{b-a}\right)^{\alpha}\left(\frac{c d}{d-c}\right)^{\beta}\left[J_{\frac{1}{b^{+}, \frac{1}{d^{+}}}}^{\alpha, \beta}(f \circ g)\left(\frac{1}{a^{\prime}}, \frac{1}{c}\right)\right. \\
\left.+J_{\frac{1}{b^{+}, \frac{1}{c^{-}}}}^{\alpha, \beta}(f \circ g)\left(\frac{1}{a}, \frac{1}{d}\right)+J_{\frac{1}{a^{-}, \frac{1}{d^{+}}}}^{\alpha, \beta}(f \circ g)\left(\frac{1}{b}, \frac{1}{c}\right)+J_{\frac{1}{a^{-}, \frac{1}{c^{-}}}}^{\alpha, \beta}(f \circ g)\left(\frac{1}{b}, \frac{1}{d}\right)\right] \\
\leqslant \frac{f(a, c)+f(a, d)+f(b, c)+f(b, d)}{4} .
\end{gathered}
$$

On summation of inequalities (3.2) and (3.3), the proof is complete.

Now utilizing Lemmas 2.1 and 1.6, we prove our coming results.

Theorem 3.2. Let $\mathrm{f}: \Delta \subset(0, \infty) \times(0, \infty) \rightarrow \mathbb{R}$ be partial differentiable function on $\Delta$ with $\mathrm{a}<\mathrm{b}$ and $\mathrm{c}<\mathrm{d}$. If $\frac{\partial^{2} \mathrm{f}}{\partial \mathrm{t} \partial \mathrm{r}} \in \mathscr{L}(\Delta)$ and $\left|\frac{\partial^{2} \mathrm{f}}{\partial \mathrm{t} \partial \mathrm{r}}\right|^{\mathrm{q}}$ where $\mathrm{q} \geqslant 1$ is coordinated harmonic convex function, then

$$
\begin{aligned}
\Xi_{\mathrm{f}}(\Delta ; \Gamma ; \alpha, \beta) \leqslant & \frac{\mathrm{ab}(\mathrm{b}-\mathrm{a}) \mathrm{cd}(\mathrm{d}-\mathrm{c})}{4} \phi^{1-\frac{1}{\mathrm{q}}}(\Delta ; \alpha ; \beta) \\
& \times\left\{\phi_{1}(\Delta ; \alpha ; \beta)\left|\frac{\partial^{2} \mathrm{f}}{\partial \mathrm{t} \partial \mathrm{r}}(\mathrm{a}, \mathrm{c})\right|^{\mathrm{q}}+\phi_{2}(\Delta ; \alpha ; \beta)\left|\frac{\partial^{2} \mathrm{f}}{\partial \mathrm{t} \partial \mathrm{r}}(\mathrm{a}, \mathrm{d})\right|^{\mathrm{q}}\right. \\
& \left.+\phi_{3}(\Delta ; \alpha ; \beta)\left|\frac{\partial^{2} \mathrm{f}}{\partial \mathrm{t} \partial \mathrm{r}}(\mathrm{b}, \mathrm{c})\right|^{\mathrm{q}}+\phi_{4}(\Delta ; \alpha ; \beta)\left|\frac{\partial^{2} \mathrm{f}}{\partial \mathrm{t} \partial \mathrm{r}}(\mathrm{b}, \mathrm{d})\right|^{\mathrm{q}}\right\}^{\frac{1}{q}},
\end{aligned}
$$

where

$$
\begin{gathered}
\phi^{1-\frac{1}{q}}(\Delta ; \alpha ; \beta)=\left\{\frac{b^{-2}}{\alpha+1}\left[{ }_{2} F_{1}\left(2,1 ; \alpha+2 ; 1-\frac{a}{b}\right)+{ }_{2} F_{1}\left(2, \alpha+1 ; \alpha+2 ; 1-\frac{a}{b}\right)\right]\right\} \\
\times\left\{\frac{d^{-2}}{\beta+1}\left[{ }_{2} F_{1}\left(2,1 ; \beta+2 ; 1-\frac{c}{d}\right)+{ }_{2} F_{1}\left(2, \beta+1 ; \beta+2 ; 1-\frac{c}{d}\right)\right]\right\}, \\
\phi_{1}(\Delta ; \alpha ; \beta)=\left\{\frac{b^{-2}}{\alpha+1}\left[{ }_{2} F_{1}\left(2,1 ; \alpha+3 ; 1-\frac{a}{b}\right)+\frac{1}{\alpha+1}{ }_{2} F_{1}\left(2, \alpha+1 ; \alpha+3 ; 1-\frac{a}{b}\right)\right]\right\} \\
\times\left\{\frac{d^{-2}}{\beta+1}\left[{ }_{2} F_{1}\left(2,1 ; \beta+3 ; 1-\frac{c}{d}\right)+\frac{1}{\beta+1}{ }_{2} F_{1}\left(2, \beta+1 ; \beta+3 ; 1-\frac{c}{d}\right)\right]\right\}, \\
\phi_{2}(\Delta ; \alpha ; \beta)=\left\{\frac{b^{-2}}{\alpha+1}\left[{ }_{2} F_{1}\left(2,1 ; \alpha+3 ; 1-\frac{a}{b}\right)+\frac{1}{\alpha+1}{ }_{2} F_{1}\left(2, \alpha+1 ; \alpha+3 ; 1-\frac{a}{b}\right)\right]\right\}
\end{gathered}
$$




$$
\begin{aligned}
\times & \left\{\frac{d^{-2}}{\beta+2}\left[\frac{1}{\beta+1}{ }_{2} F_{1}\left(2,2 ; \beta+3 ; 1-\frac{c}{d}\right)+{ }_{2} F_{1}\left(2, \beta+2 ; \beta+3 ; 1-\frac{c}{d}\right)\right]\right\}, \\
\phi_{3}(\Delta ; \alpha ; \beta)= & \left\{\frac{b^{-2}}{\alpha+2}\left[\frac{1}{\alpha+1}{ }_{2} F_{1}\left(2,2 ; \alpha+3 ; 1-\frac{a}{b}\right)+{ }_{2} F_{1}\left(2, \alpha+2 ; \alpha+3 ; 1-\frac{a}{b}\right)\right]\right\} \\
& \times\left\{\frac{d^{-2}}{\beta+1}\left[{ }_{2} F_{1}\left(2,1 ; \beta+3 ; 1-\frac{c}{d}\right)+\frac{1}{\beta+1}{ }_{2} F_{1}\left(2, \beta+1 ; \beta+3 ; 1-\frac{c}{d}\right)\right]\right\},
\end{aligned}
$$

and

$$
\begin{aligned}
\phi_{4}(\Delta ; \alpha ; \beta)= & \left\{\frac{\mathrm{b}^{-2}}{\alpha+2}\left[\frac{1}{\alpha+1}{ }_{2} \mathrm{~F}_{1}\left(2,2 ; \alpha+3 ; 1-\frac{\mathrm{a}}{\mathrm{b}}\right)+{ }_{2} \mathrm{~F}_{1}\left(2, \alpha+2 ; \alpha+3 ; 1-\frac{\mathrm{a}}{\mathrm{b}}\right)\right]\right\} \\
& \times\left\{\frac{\mathrm{d}^{-2}}{\beta+2}\left[\frac{1}{\beta+1}{ }_{2} \mathrm{~F}_{1}\left(2,2 ; \beta+3 ; 1-\frac{\mathrm{c}}{\mathrm{d}}\right)+{ }_{2} \mathrm{~F}_{1}\left(2, \beta+2 ; \beta+3 ; 1-\frac{\mathrm{c}}{\mathrm{d}}\right)\right]\right\} .
\end{aligned}
$$

Proof. Using Lemma 2.1, property of the modulus, power mean's inequality and the fact that $\left|\frac{\partial^{2} f}{\partial t \partial r}\right|^{q}$ is coordinated harmonic convex function, we have

$$
\begin{aligned}
& \left|\Xi_{f}(\Delta ; \Gamma ; \alpha, \beta)\right|=\mid \frac{a b(b-a) c d(d-c)}{4} \\
& \times\left\{\int_{0}^{1} \int_{0}^{1}\left(\frac{t^{\alpha}-(1-t)^{\alpha}}{A_{t}^{2}}\right)\left(\frac{r^{\beta}-(1-r)^{\beta}}{A_{r}^{2}}\right) \frac{\partial^{2} f}{\partial t \partial r}\left(\frac{a b}{A_{t}}, \frac{c d}{A_{r}}\right) d r d t\right\} \mid \\
& \leqslant \frac{a b(b-a) c d(d-c)}{4} \\
& \times\left\{\int_{0}^{1} \int_{0}^{1}\left(\frac{\left|t^{\alpha}-(1-t)^{\alpha}\right|}{A_{t}^{2}}\right)\left(\frac{\left|r^{\beta}-(1-r)^{\beta}\right|}{A_{r}^{2}}\right)\left|\frac{\partial^{2} f}{\partial t \partial r}\left(\frac{a b}{A_{t}}, \frac{c d}{A_{r}}\right)\right| d r d t\right\} \\
& \leqslant \frac{a b(b-a) c d(d-c)}{4} \\
& \times\left(\int_{0}^{1} \int_{0}^{1}\left(\frac{\left|(1-t)^{\alpha}-t^{\alpha}\right|}{A_{t}^{2}}\right)\left(\frac{\left|(1-r)^{\beta}-r^{\beta}\right|}{A_{r}^{2}}\right) d r d t\right)^{1-\frac{1}{q}} \\
& \times\left(\int_{0}^{1} \int_{0}^{1}\left(\frac{\left|(1-t)^{\alpha}-t^{\alpha}\right|}{A_{t}^{2}}\right)\left(\frac{\left|(1-r)^{\beta}-r^{\beta}\right|}{A_{r}^{2}}\right)\left|\frac{\partial^{2} f}{\partial t \partial r}\left(\frac{a b}{A_{t}}, \frac{c d}{A_{r}}\right)\right|^{q} d r d t\right)^{\frac{1}{q}} \\
& \leqslant \frac{a b(b-a) c d(d-c)}{4} \\
& \times\left(\int_{0}^{1} \int_{0}^{1}\left(\frac{\left[(1-t)^{\alpha}+t^{\alpha}\right]}{A_{t}^{2}}\right)\left(\frac{\left[(1-r)^{\beta}+r^{\beta}\right]}{A_{r}^{2}}\right) d r d t\right)^{1-\frac{1}{q}} \\
& \times\left\{\int_{0}^{1} \int_{0}^{1}\left(\frac{\left[(1-t)^{\alpha}+t^{\alpha}\right]}{A_{t}^{2}}\right)\left(\frac{\left[(1-r)^{\beta}+r^{\beta}\right]}{A_{r}^{2}}\right)\right.
\end{aligned}
$$




$$
\begin{aligned}
& \quad \times\left[(1-t)(1-r)\left|\frac{\partial^{2} f}{\partial t \partial r}(a, c)\right|^{q}+(1-t) r\left|\frac{\partial^{2} f}{\partial t \partial r}(a, d)\right|^{q}\right. \\
& \left.\left.+t(1-r)\left|\frac{\partial^{2} f}{\partial t \partial r}(b, c)\right|^{q}+\operatorname{tr}\left|\frac{\partial^{2} f}{\partial t \partial r}(b, d)\right|\right] d r d t\right\}^{\frac{1}{q}} \\
& =\frac{a b(b-a) c d(d-c)}{4} \phi^{1-\frac{1}{q}}(\Delta ; \alpha ; \beta) \\
& \times\left\{\phi_{1}(\Delta ; \alpha ; \beta)\left|\frac{\partial^{2} f}{\partial t \partial r}(a, c)\right|^{q}+\phi_{2}(\Delta ; \alpha ; \beta)\left|\frac{\partial^{2} f}{\partial t \partial r}(a, d)\right|^{q}\right. \\
& \left.+\phi_{3}(\Delta ; \alpha ; \beta)\left|\frac{\partial^{2} f}{\partial t \partial r}(b, c)\right|^{q}+\phi_{4}(\Delta ; \alpha ; \beta)\left|\frac{\partial^{2} f}{\partial t \partial r}(b, d)\right|^{q}\right\}^{\frac{1}{q}},
\end{aligned}
$$

where

$$
\begin{aligned}
& \phi(\Delta ; \alpha ; \beta)=\int_{0}^{1} \int_{0}^{1}\left(\frac{\left[(1-t)^{\alpha}+t^{\alpha}\right]}{A_{t}^{2}}\right)\left(\frac{\left[(1-r)^{\beta}+r^{\beta}\right]}{A_{r}^{2}}\right) d r d t \\
& =\left\{\frac{b^{-2}}{\alpha+1}\left[{ }_{2} F_{1}\left(2,1 ; \alpha+2 ; 1-\frac{a}{b}\right)+{ }_{2} F_{1}\left(2, \alpha+1 ; \alpha+2 ; 1-\frac{a}{b}\right)\right]\right\} \\
& \times\left\{\frac{d^{-2}}{\beta+1}\left[{ }_{2} F_{1}\left(2,1 ; \beta+2 ; 1-\frac{c}{d}\right)+{ }_{2} F_{1}\left(2, \beta+1 ; \beta+2 ; 1-\frac{c}{d}\right)\right]\right\}, \\
& \phi_{1}(\Delta ; \alpha ; \beta)=\int_{0}^{1} \int_{0}^{1}(1-t)(1-r)\left(\frac{\left[(1-t)^{\alpha}+t^{\alpha}\right]}{A_{t}^{2}}\right)\left(\frac{\left[(1-r)^{\beta}+r^{\beta}\right]}{A_{r}^{2}}\right) d r d t \\
& =\left\{\frac{b^{-2}}{\alpha+1}\left[{ }_{2} F_{1}\left(2,1 ; \alpha+3 ; 1-\frac{a}{b}\right)+\frac{1}{\alpha+1}{ }_{2} F_{1}\left(2, \alpha+1 ; \alpha+3 ; 1-\frac{a}{b}\right)\right]\right\} \\
& \times\left\{\frac{d^{-2}}{\beta+1}\left[{ }_{2} F_{1}\left(2,1 ; \beta+3 ; 1-\frac{c}{d}\right)+\frac{1}{\beta+1}{ }_{2} F_{1}\left(2, \beta+1 ; \beta+3 ; 1-\frac{c}{d}\right)\right]\right\}, \\
& \phi_{2}(\Delta ; \alpha ; \beta)=\int_{0}^{1} \int_{0}^{1}(1-t) r\left(\frac{\left[(1-t)^{\alpha}+t^{\alpha}\right]}{A_{t}^{2}}\right)\left(\frac{\left[(1-r)^{\beta}+r^{\beta}\right]}{A_{r}^{2}}\right) d r d t \\
& =\left\{\frac{b^{-2}}{\alpha+1}\left[{ }_{2} F_{1}\left(2,1 ; \alpha+3 ; 1-\frac{a}{b}\right)+\frac{1}{\alpha+1}{ }_{2} F_{1}\left(2, \alpha+1 ; \alpha+3 ; 1-\frac{a}{b}\right)\right]\right\} \\
& \times\left\{\frac{d^{-2}}{\beta+2}\left[\frac{1}{\beta+1}{ }_{2} F_{1}\left(2,2 ; \beta+3 ; 1-\frac{c}{d}\right)+{ }_{2} F_{1}\left(2, \beta+2 ; \beta+3 ; 1-\frac{c}{d}\right)\right]\right\}, \\
& \phi_{3}(\Delta ; \alpha ; \beta)=\int_{0}^{1} \int_{0}^{1} t(1-r)\left(\frac{\left[(1-t)^{\alpha}+t^{\alpha}\right]}{A_{t}^{2}}\right)\left(\frac{\left[(1-r)^{\beta}+r^{\beta}\right]}{A_{r}^{2}}\right) d r d t \\
& =\left\{\frac{b^{-2}}{\alpha+2}\left[\frac{1}{\alpha+1}{ }_{2} F_{1}\left(2,2 ; \alpha+3 ; 1-\frac{a}{b}\right)+{ }_{2} F_{1}\left(2, \alpha+2 ; \alpha+3 ; 1-\frac{a}{b}\right)\right]\right\}
\end{aligned}
$$




$$
\times\left\{\frac{d^{-2}}{\beta+1}\left[{ }_{2} F_{1}\left(2,1 ; \beta+3 ; 1-\frac{c}{d}\right)+\frac{1}{\beta+1}{ }_{2} F_{1}\left(2, \beta+1 ; \beta+3 ; 1-\frac{c}{d}\right)\right]\right\}
$$

and

$$
\begin{aligned}
\phi_{4}(\Delta ; \alpha ; \beta)= & \int_{0}^{1} \int_{0}^{1} \operatorname{tr}\left(\frac{\left[(1-\mathrm{t})^{\alpha}+\mathrm{t}^{\alpha}\right]}{\mathrm{A}_{\mathrm{t}}^{2}}\right)\left(\frac{\left[(1-\mathrm{r})^{\beta}+\mathrm{r}^{\beta}\right]}{\mathrm{A}_{\mathrm{r}}^{2}}\right) \mathrm{drdt} \\
= & \left\{\frac{\mathrm{b}^{-2}}{\alpha+2}\left[\frac{1}{\alpha+1}{ }_{2} \mathrm{~F}_{1}\left(2,2 ; \alpha+3 ; 1-\frac{\mathrm{a}}{\mathrm{b}}\right)+{ }_{2} \mathrm{~F}_{1}\left(2, \alpha+2 ; \alpha+3 ; 1-\frac{\mathrm{a}}{\mathrm{b}}\right)\right]\right\} \\
& \times\left\{\frac{\mathrm{d}^{-2}}{\beta+2}\left[\frac{1}{\beta+1}{ }_{2} \mathrm{~F}_{1}\left(2,2 ; \beta+3 ; 1-\frac{\mathrm{c}}{\mathrm{d}}\right)+{ }_{2} \mathrm{~F}_{1}\left(2, \beta+2 ; \beta+3 ; 1-\frac{\mathrm{c}}{\mathrm{d}}\right)\right]\right\} .
\end{aligned}
$$

This completes the proof.

Theorem 3.3. Let $\mathrm{f}: \Delta \subset(0, \infty) \times(0, \infty) \rightarrow \mathbb{R}$ be partial differentiable function on $\Delta$ with $\mathrm{a}<\mathrm{b}$ and $\mathrm{c}<\mathrm{d}$. If $\frac{\partial^{2} \mathrm{f}}{\partial \mathrm{t} \partial \mathrm{r}} \in \mathscr{L}(\Delta)$ and $\left|\frac{\partial^{2} \mathrm{f}}{\partial \mathrm{t} \partial \mathrm{r}}\right|^{\mathrm{q}}$ where $\mathrm{q} \geqslant 1$ is coordinated harmonic convex function, then

$$
\begin{aligned}
\Xi_{\mathrm{f}}(\Delta ; \Gamma ; \alpha, \beta) \leqslant & \frac{\mathrm{ab}(\mathrm{b}-\mathrm{a}) \mathrm{cd}(\mathrm{d}-\mathrm{c})}{4} \psi^{1-\frac{1}{\mathrm{q}}}(\Delta ; \alpha ; \beta) \\
& \times\left\{\psi_{1}(\Delta ; \alpha ; \beta)\left|\frac{\partial^{2} \mathrm{f}}{\partial \mathrm{t} \partial \mathrm{r}}(\mathrm{a}, \mathrm{c})\right|^{\mathrm{q}}+\psi_{2}(\Delta ; \alpha ; \beta)\left|\frac{\partial^{2} \mathrm{f}}{\partial \mathrm{t} \partial \mathrm{r}}(\mathrm{a}, \mathrm{d})\right|^{\mathrm{q}}\right. \\
& \left.+\psi_{3}(\Delta ; \alpha ; \beta)\left|\frac{\partial^{2} \mathrm{f}}{\partial \mathrm{t} \partial \mathrm{r}}(\mathrm{b}, \mathrm{c})\right|^{\mathrm{q}}+\psi_{4}(\Delta ; \alpha ; \beta)\left|\frac{\partial^{2} \mathrm{f}}{\partial \mathrm{t} \partial \mathrm{r}}(\mathrm{b}, \mathrm{d})\right|^{\mathrm{q}}\right\}^{\frac{1}{q}},
\end{aligned}
$$

where

$$
\begin{aligned}
& \psi^{1-\frac{1}{q}}(\Delta ; \alpha ; \beta)=\left\{\frac { b ^ { - 2 } } { \alpha + 1 } \left[{ }_{2} F_{1}\left(2,1 ; \alpha+1 ; 1-\frac{a}{b}\right)+{ }_{2} F_{1}\left(2,1 ; \alpha+2 ; 1-\frac{a}{b}\right)\right.\right. \\
& \left.\left.+{ }_{2} \mathrm{~F}_{1}\left(2,1 ; \alpha+2 ; \frac{1}{2}\left(1-\frac{\mathrm{c}}{\mathrm{d}}\right)\right)\right]\right\} \times\left\{\frac { \mathrm { d } ^ { - 2 } } { \beta + 1 } \left[{ }_{2} \mathrm{~F}_{1}\left(2,1 ; \beta+1 ; 1-\frac{\mathrm{c}}{\mathrm{d}}\right)\right.\right. \\
& \left.\left.+{ }_{2} \mathrm{~F}_{1}\left(2,1 ; \beta+2 ; 1-\frac{\mathrm{c}}{\mathrm{d}}\right)+{ }_{2} \mathrm{~F}_{1}\left(2,1 ; \beta+2 ; \frac{1}{2}\left(1-\frac{\mathrm{c}}{\mathrm{d}}\right)\right)\right]\right\} \\
& \psi_{1}(\Delta ; \alpha ; \beta)=\left\{\frac { b ^ { - 2 } } { \alpha + 2 } \left[\frac{1}{\alpha+1}{ }_{2} F_{1}\left(2, \alpha+1 ; \alpha+3 ; 1-\frac{a}{b}\right)-{ }_{2} F_{1}\left(2,1 ; \alpha+3 ; 1-\frac{a}{b}\right)\right.\right. \\
& \left.\left.+{ }_{2} \mathrm{~F}_{1}\left(2,1 ; \alpha+3 ; \frac{1}{2}\left(1-\frac{\mathrm{a}}{\mathrm{b}}\right)\right)\right]\right\} \times\left\{\frac { \mathrm { d } ^ { - 2 } } { \beta + 2 } \left[{ }_{2} \mathrm{~F}_{1}\left(2, \beta+1 ; \beta+3 ; 1-\frac{\mathrm{c}}{\mathrm{d}}\right)\right.\right. \\
& \left.\left.+{ }_{2} \mathrm{~F}_{1}\left(2,1 ; \beta+3 ; 1-\frac{\mathrm{c}}{\mathrm{d}}\right)+{ }_{2} \mathrm{~F}_{1}\left(2,1 ; \beta+3 ; \frac{1}{2}\left(1-\frac{\mathrm{a}}{\mathrm{b}}\right)\right)\right]\right\} \text {, } \\
& \psi_{2}(\Delta ; \alpha ; \beta)=\left\{\frac { b ^ { - 2 } } { \alpha + 2 } \left[\frac{1}{\alpha+1}{ }_{2} F_{1}\left(2, \alpha+1 ; \alpha+3 ; 1-\frac{a}{b}\right)-{ }_{2} F_{1}\left(2,1 ; \alpha+3 ; 1-\frac{a}{b}\right)\right.\right.
\end{aligned}
$$




$$
\begin{aligned}
&\left.\left.+{ }_{2} \mathrm{~F}_{1}\left(2,1 ; \alpha+3 ; \frac{1}{2}\left(1-\frac{\mathrm{a}}{\mathrm{b}}\right)\right)\right]\right\} \times\left\{\frac { \mathrm { d } ^ { - 2 } } { \beta + 2 } \left[{ }_{2} \mathrm{~F}_{1}\left(2, \beta+2 ; \beta+3 ; 1-\frac{\mathrm{c}}{\mathrm{d}}\right)\right.\right. \\
&\left.\left.-\frac{1}{\beta+1}{ }_{2} \mathrm{~F}_{1}\left(2,2 ; \beta+3 ; 1-\frac{\mathrm{c}}{\mathrm{d}}\right)+\frac{1}{2(\beta+1)}{ }_{2} \mathrm{~F}_{1}\left(2,2 ; \beta+3 ; \frac{1}{2}\left(1-\frac{\mathrm{a}}{\mathrm{b}}\right)\right)\right]\right\}, \\
& \psi_{3}(\Delta ; \alpha ; \beta)=\left\{\frac { \mathrm { b } ^ { - 2 } } { \alpha + 2 } \left[{ }_{2} \mathrm{~F}_{1}\left(2, \alpha+2 ; \alpha+3 ; 1-\frac{\mathrm{a}}{\mathrm{b}}\right)-\frac{1}{\alpha+1}{ }_{2} \mathrm{~F}_{1}\left(2,2 ; \alpha+3 ; 1-\frac{\mathrm{a}}{\mathrm{b}}\right)\right.\right. \\
&+\left.\left.\frac{1}{2(\alpha+1)}{ }_{2} \mathrm{~F}_{1}\left(2,2 ; \alpha+3 ; \frac{1}{2}\left(1-\frac{\mathrm{a}}{\mathrm{b}}\right)\right)\right]\right\} \times\left\{\frac { \mathrm { d } ^ { - 2 } } { \beta + 2 } \left[{ }_{2} \mathrm{~F}_{1}\left(2, \beta+1 ; \beta+3 ; 1-\frac{\mathrm{c}}{\mathrm{d}}\right)\right.\right. \\
&\left.\left.+{ }_{2} \mathrm{~F}_{1}\left(2,1 ; \beta+3 ; 1-\frac{\mathrm{c}}{\mathrm{d}}\right)+{ }_{2} \mathrm{~F}_{1}\left(2,1 ; \beta+3 ; \frac{1}{2}\left(1-\frac{\mathrm{a}}{\mathrm{b}}\right)\right)\right]\right\},
\end{aligned}
$$

and

$$
\begin{aligned}
\psi_{4}(\Delta ; \alpha ; \beta)= & \frac{b^{-2}}{\alpha+2}\left[{ }_{2} \mathrm{~F}_{1}\left(2, \alpha+2 ; \alpha+3 ; 1-\frac{\mathrm{a}}{\mathrm{b}}\right)-\frac{1}{\alpha+1}{ }_{2} \mathrm{~F}_{1}\left(2,2 ; \alpha+3 ; 1-\frac{\mathrm{a}}{\mathrm{b}}\right)\right. \\
& \left.\left.+\frac{1}{2(\alpha+1)}{ }_{2} \mathrm{~F}_{1}\left(2,2 ; \alpha+3 ; \frac{1}{2}\left(1-\frac{\mathrm{a}}{\mathrm{b}}\right)\right)\right]\right\} \times\left\{\frac { \mathrm { d } ^ { - 2 } } { \beta + 2 } \left[{ }_{2} \mathrm{~F}_{1}\left(2, \beta+2 ; \beta+3 ; 1-\frac{\mathrm{c}}{\mathrm{d}}\right)\right.\right. \\
& \left.\left.-\frac{1}{\beta+1}{ }_{2} \mathrm{~F}_{1}\left(2,2 ; \beta+3 ; 1-\frac{\mathrm{c}}{\mathrm{d}}\right)+\frac{1}{2(\beta+1)}{ }_{2} \mathrm{~F}_{1}\left(2,2 ; \beta+3 ; \frac{1}{2}\left(1-\frac{\mathrm{a}}{\mathrm{b}}\right)\right)\right]\right\} .
\end{aligned}
$$

Proof. Using Lemma 2.1, property of the modulus, power mean's inequality and the fact that $\left|\frac{\partial^{2} f}{\partial t \partial r}\right|^{q}$ is coordinated harmonic convex function, we have

$$
\begin{aligned}
\left|\Xi_{f}(\Delta ; \Gamma ; \alpha, \beta)\right|= & \mid \frac{a b(b-a) c d(d-c)}{4} \\
& \times\left\{\iint_{0}^{1} \int_{0}^{1}\left(\frac{t^{\alpha}-(1-t)^{\alpha}}{A_{t}^{2}}\right)\left(\frac{r^{\beta}-(1-r)^{\beta}}{A_{r}^{2}}\right) \frac{\partial^{2} f}{\partial t \partial r}\left(\frac{a b}{A_{t}}, \frac{c d}{A_{r}}\right) d r d t\right\} \mid \\
\leqslant & \frac{a b(b-a) c d(d-c)}{4} \\
& \times\left\{\int_{0}^{1} \int_{0}^{1}\left(\frac{\left|t^{\alpha}-(1-t)^{\alpha}\right|}{A_{t}^{2}}\right)\left(\frac{\left|r^{\beta}-(1-r)^{\beta}\right|}{A_{r}^{2}}\right)\left|\frac{\partial^{2} f}{\partial t \partial r}\left(\frac{a b}{A_{t}}, \frac{c d}{A_{r}}\right)\right| d r d t\right\} \\
\leqslant & \left.\left.\frac{a b(b-a) c d(d-c)}{4}\right)\left(\frac{\left|(1-r)^{\beta}-r^{\beta}\right|}{A_{r}^{2}}\right) d r d t\right)^{1-\frac{1}{q}} \\
& \times\left(\int_{0}^{1} \int_{0}^{1}\left(\frac{\left|(1-t)^{\alpha}-t^{\alpha}\right|}{A_{t}^{2}}\right)\right. \\
& \times\left(\int_{0}^{1} \int_{0}^{1}\left(\frac{\left|(1-t)^{\alpha}-t^{\alpha}\right|}{A_{t}^{2}}\right)\left(\frac{\left|(1-r)^{\beta}-r^{\beta}\right|}{A_{r}^{2}}\right)\left|\frac{\partial^{2} f}{\partial t \partial r}\left(\frac{a b}{A_{t}}, \frac{c d}{A_{r}}\right)\right|^{q} d r d t\right)^{\frac{1}{q}} \\
\leqslant & \frac{a b(b-a) c d(d-c)}{4}
\end{aligned}
$$




$$
\begin{aligned}
& \times\left(\int_{0}^{1} \int_{0}^{1}\left(\frac{\left|(1-t)^{\alpha}-t^{\alpha}\right|}{A_{t}^{2}}\right)\left(\frac{\left|(1-r)^{\beta}-r^{\beta}\right|}{A_{r}^{2}}\right) d r d t\right)^{1-\frac{1}{q}} \\
& \times\left\{\iint_{0}^{1}\left(\frac{\left|(1-r)^{\alpha}-r^{\alpha}\right|}{A_{t}^{2}}\right)\left(\frac{\left|(1-r)^{\beta}-r^{\beta}\right|}{A_{r}^{2}}\right)\right. \\
& \times\left[(1-t)(1-r)\left|\frac{\partial^{2} f}{\partial t \partial r}(a, c)\right|^{q}+(1-t) r\left|\frac{\partial^{2} f}{\partial t \partial r}(a, d)\right|^{q}\right. \\
& \left.\left.+t(1-r)\left|\frac{\partial^{2} f}{\partial t \partial r}(b, c)\right|^{q}+t r\left|\frac{\partial^{2} f}{\partial t \partial r}(b, d)\right|\right] d r d t\right\}^{\frac{1}{q}} \\
& =\frac{a b(b-a) c d(d-c)}{4} \psi^{1-\frac{1}{q}}(\Delta ; \alpha ; \beta) \\
& \times\left\{\psi_{1}(\Delta ; \alpha ; \beta)\left|\frac{\partial^{2} f}{\partial t \partial r}(a, c)\right|^{q}+\psi_{2}(\Delta ; \alpha ; \beta)\left|\frac{\partial^{2} f}{\partial t \partial r}(a, d)\right|^{q}\right. \\
& \left.+\psi_{3}(\Delta ; \alpha ; \beta)\left|\frac{\partial^{2} f}{\partial t \partial r}(b, c)\right|^{q}+\psi_{4}(\Delta ; \alpha ; \beta)\left|\frac{\partial^{2} f}{\partial t \partial r}(b, d)\right|^{q}\right\}^{\frac{1}{q}},
\end{aligned}
$$

where

$$
\begin{aligned}
& \psi(\Delta ; \alpha ; \beta)= \int_{0}^{1} \int_{0}^{1}\left(\frac{\left|(1-r)^{\alpha}-r^{\alpha}\right|}{A_{t}^{2}}\right)\left(\frac{\left|(1-r)^{\beta}-r^{\beta}\right|}{A_{r}^{2}}\right) d r d t \\
&=\left\{\frac { b ^ { - 2 } } { \alpha + 1 } \left[{ }_{2} F_{1}\left(2,1 ; \alpha+1 ; 1-\frac{a}{b}\right)+{ }_{2} F_{1}\left(2,1 ; \alpha+2 ; 1-\frac{a}{b}\right)\right.\right. \\
&\left.\left.+{ }_{2} F_{1}\left(2,1 ; \alpha+2 ; \frac{1}{2}\left(1-\frac{c}{d}\right)\right)\right]\right\} \times\left\{\frac { d ^ { - 2 } } { \beta + 1 } \left[{ }_{2} F_{1}\left(2,1 ; \beta+1 ; 1-\frac{c}{d}\right)\right.\right. \\
&\left.\left.+{ }_{2} F_{1}\left(2,1 ; \beta+2 ; 1-\frac{c}{d}\right)+{ }_{2} F_{1}\left(2,1 ; \beta+2 ; \frac{1}{2}\left(1-\frac{c}{d}\right)\right)\right]\right\}, \\
& \psi_{1}(\Delta ; \alpha ; \beta)= \int_{0}^{1} \int_{0}^{1}(1-t)(1-r)\left(\frac{\left[(1-t)^{\alpha}+t^{\alpha}\right]}{A_{t}^{2}}\right)\left(\frac{\left[(1-r)^{\beta}+r^{\beta}\right]}{A_{r}^{2}}\right) d r d t \\
&=\{ \frac{b^{-2}}{\alpha+2}\left[\frac{1}{\alpha+1}{ }_{2} F_{1}\left(2, \alpha+1 ; \alpha+3 ; 1-\frac{a}{b}\right)-{ }_{2} F_{1}\left(2,1 ; \alpha+3 ; 1-\frac{a}{b}\right)\right. \\
&\left.\left.+{ }_{2} F_{1}\left(2,1 ; \alpha+3 ; \frac{1}{2}\left(1-\frac{a}{b}\right)\right)\right]\right\} \times\left\{\frac { d ^ { - 2 } } { \beta + 2 } \left[{ }_{2} F_{1}\left(2, \beta+1 ; \beta+3 ; 1-\frac{c}{d}\right)\right.\right. \\
&\left.\left.+{ }_{2} F_{1}\left(2,1 ; \beta+3 ; 1-\frac{c}{d}\right)+{ }_{2} F_{1}\left(2,1 ; \beta+3 ; \frac{1}{2}\left(1-\frac{a}{b}\right)\right)\right]\right\}, \\
& \Psi_{2}(\Delta ; \alpha ; \beta)=\int_{0}^{1} \int_{0}^{1}(1-t) r\left(\frac{\left[(1-t)^{\alpha}+t^{\alpha}\right]}{A_{t}^{2}}\right)\left(\frac{[(1-r)}{A_{r}^{2}}\right) d r d t
\end{aligned}
$$




$$
\begin{aligned}
= & \left\{\frac { b ^ { - 2 } } { \alpha + 2 } \left[\frac{1}{\alpha+1}{ }_{2} F_{1}\left(2, \alpha+1 ; \alpha+3 ; 1-\frac{a}{b}\right)-{ }_{2} F_{1}\left(2,1 ; \alpha+3 ; 1-\frac{a}{b}\right)\right.\right. \\
& \left.\left.+{ }_{2} F_{1}\left(2,1 ; \alpha+3 ; \frac{1}{2}\left(1-\frac{a}{b}\right)\right)\right]\right\} \times\left\{\frac { d ^ { - 2 } } { \beta + 2 } \left[{ }_{2} F_{1}\left(2, \beta+2 ; \beta+3 ; 1-\frac{c}{d}\right)\right.\right. \\
& \left.\left.-\frac{1}{\beta+1}{ }_{2} F_{1}\left(2,2 ; \beta+3 ; 1-\frac{c}{d}\right)+\frac{1}{2(\beta+1)}{ }_{2} F_{1}\left(2,2 ; \beta+3 ; \frac{1}{2}\left(1-\frac{a}{b}\right)\right)\right]\right\}, \\
\psi_{3}(\Delta ; \alpha ; \beta)= & \int_{0}^{1} \int_{0}^{1} t(1-r)\left(\frac{\left[(1-t)^{\alpha}+t^{\alpha}\right]}{A_{t}^{2}}\right)\left(\frac{\left[(1-r){ }^{\beta}+r^{\beta}\right]}{A_{r}^{2}}\right) d r d t \\
=\{ & \frac{b^{-2}}{\alpha+2}\left[{ }_{2} F_{1}\left(2, \alpha+2 ; \alpha+3 ; 1-\frac{a}{b}\right)-\frac{1}{\alpha+1}{ }_{2} F_{1}\left(2,2 ; \alpha+3 ; 1-\frac{a}{b}\right)\right. \\
& \left.\left.+\frac{1}{2(\alpha+1)}{ }_{2} F_{1}\left(2,2 ; \alpha+3 ; \frac{1}{2}\left(1-\frac{a}{b}\right)\right)\right]\right\} \times\left\{\frac { d ^ { - 2 } } { \beta + 2 } \left[{ }_{2} F_{1}\left(2, \beta+1 ; \beta+3 ; 1-\frac{c}{d}\right)\right.\right. \\
& \left.\left.+{ }_{2} F_{1}\left(2,1 ; \beta+3 ; 1-\frac{c}{d}\right)+{ }_{2} F_{1}\left(2,1 ; \beta+3 ; \frac{1}{2}\left(1-\frac{a}{b}\right)\right)\right]\right\},
\end{aligned}
$$

and

$$
\begin{aligned}
\psi_{4}(\Delta ; \alpha ; \beta)= & \int_{0}^{1} \int_{0}^{1} \operatorname{tr}\left(\frac{\left[(1-\mathrm{t})^{\alpha}+\mathrm{t}^{\alpha}\right]}{\mathrm{A}_{\mathrm{t}}^{2}}\right)\left(\frac{\left[(1-\mathrm{r})^{\beta}+\mathrm{r}^{\beta}\right]}{\mathrm{A}_{\mathrm{r}}^{2}}\right) \mathrm{drdt} \\
= & \left\{\frac { \mathrm { b } ^ { - 2 } } { \alpha + 2 } \left[{ }_{2} \mathrm{~F}_{1}\left(2, \alpha+2 ; \alpha+3 ; 1-\frac{\mathrm{a}}{\mathrm{b}}\right)-\frac{1}{\alpha+1}{ }_{2} \mathrm{~F}_{1}\left(2,2 ; \alpha+3 ; 1-\frac{\mathrm{a}}{\mathrm{b}}\right)\right.\right. \\
& \left.\left.+\frac{1}{2(\alpha+1)}{ }_{2} \mathrm{~F}_{1}\left(2,2 ; \alpha+3 ; \frac{1}{2}\left(1-\frac{\mathrm{a}}{\mathrm{b}}\right)\right)\right]\right\} \times\left\{\frac { \mathrm { d } ^ { - 2 } } { \beta + 2 } \left[{ }_{2} \mathrm{~F}_{1}\left(2, \beta+2 ; \beta+3 ; 1-\frac{\mathrm{c}}{\mathrm{d}}\right)\right.\right. \\
& \left.\left.-\frac{1}{\beta+1}{ }_{2} \mathrm{~F}_{1}\left(2,2 ; \beta+3 ; 1-\frac{\mathrm{c}}{\mathrm{d}}\right)+\frac{1}{2(\beta+1)}{ }_{2} \mathrm{~F}_{1}\left(2,2 ; \beta+3 ; \frac{1}{2}\left(1-\frac{\mathrm{a}}{\mathrm{b}}\right)\right)\right]\right\} .
\end{aligned}
$$

This completes the proof.

Theorem 3.4. Let $\mathrm{f}: \Delta \subset(0, \infty) \times(0, \infty) \rightarrow \mathbb{R}$ be partial differentiable function on $\Delta$ with $\mathrm{a}<\mathrm{b}$ and $\mathrm{c}<\mathrm{d}$. If $\frac{\partial^{2} f}{\partial t \partial r} \in \mathscr{L}(\Delta)$ and $\left|\frac{\partial^{2} f}{\partial t \partial r}\right|^{p}$ where $\frac{1}{p}+\frac{1}{q}=1$ is coordinated harmonic convex function, then

$$
\begin{aligned}
\Xi_{f}(\Delta ; \Gamma ; \alpha, \beta) \leqslant & \frac{a b(b-a) c d(d-c)}{4} \theta^{\frac{1}{p}}(\Delta ; \alpha ; \beta) \theta_{1}^{\frac{1}{q}}(\Delta ; \alpha, \beta) \\
& \times\left(\left|\frac{\partial^{2} f}{\partial t \partial r}(a, c)\right|^{q}+\left|\frac{\partial^{2} f}{\partial t \partial r}(a, d)\right|^{q}+\left|\frac{\partial^{2} f}{\partial t \partial r}(b, c)\right|^{q}+\left|\frac{\partial^{2} f}{\partial t \partial r}(b, d)\right|^{q}\right)^{\frac{1}{q}},
\end{aligned}
$$

where

$$
\theta^{\frac{1}{p}}(\Delta ; \alpha, \beta)=\left(b^{-2 p}{ }_{2} F_{1}\left(2 p, 1 ; 2 ; 1-\frac{a}{b}\right)\right) \times\left(d^{-2 p}{ }_{2} F_{1}\left(2 p, 1 ; 2 ; 1-\frac{c}{d}\right)\right),
$$

and

$$
\theta_{1}(\Delta ; \alpha, \beta)=\left(\frac{1}{2(\alpha q+1)}\right)\left(\frac{1}{2(\beta q+1)}\right)
$$


Proof. Using Lemma 2.1, property of the modulus, Hölder's inequality and the fact that $\left|\frac{\partial^{2} f}{\partial t \partial r}\right|^{p}$ is coordinated harmonic convex function, we have

$$
\begin{aligned}
& \left|\Xi_{\mathrm{f}}(\Delta ; \Gamma ; \alpha, \beta)\right|=\mid \frac{\mathrm{ab}(\mathrm{b}-\mathrm{a}) \mathrm{cd}(\mathrm{d}-\mathrm{c})}{4} \\
& \times\left\{\int_{0}^{1} \int_{0}^{1}\left(\frac{t^{\alpha}-(1-t)^{\alpha}}{A_{t}^{2}}\right)\left(\frac{r^{\beta}-(1-r)^{\beta}}{A_{r}^{2}}\right) \frac{\partial^{2} f}{\partial t \partial r}\left(\frac{a b}{A_{t}}, \frac{c d}{A_{r}}\right) d r d t\right\} \mid \\
& \leqslant \frac{a b(b-a) c d(d-c)}{4} \\
& \times\left(\int_{0}^{1} \int_{0}^{1} \frac{1}{A_{t}^{2 p} A_{r}^{2 p}} d r d t\right)^{\frac{1}{p}} \times\left(\int_{0}^{1} \int_{0}^{1}\left(\left|(1-t)^{\alpha}-t^{\alpha}\right|^{q}\right)\left(\left|(1-r)^{\beta}-r^{\beta}\right|^{q}\right)\right. \\
& \times\left[(1-t)(1-r)\left|\frac{\partial^{2} f}{\partial t \partial r}(a, c)\right|^{q}+(1-t) r\left|\frac{\partial^{2} f}{\partial t \partial r}(a, d)\right|^{q}\right. \\
& \left.\left.+t(1-r)\left|\frac{\partial^{2} f}{\partial t \partial r}(b, c)\right|^{q}+\operatorname{tr}\left|\frac{\partial^{2} f}{\partial t \partial r}(b, d)\right|^{q}\right] d r d t\right)^{\frac{1}{q}} \\
& \leqslant \frac{a b(b-a) c d(d-c)}{4} \\
& \times\left(\int_{0}^{1} \int_{0}^{1} \frac{1}{A_{t}^{2 p} A_{r}^{2 p}} d r d t\right)^{\frac{1}{p}} \times\left(\int_{0}^{1} \int_{0}^{1}\left(|1-2 t|^{q \alpha}\right)\left(|1-2 r|^{q \beta}\right)\right. \\
& \times\left[(1-t)(1-r)\left|\frac{\partial^{2} f}{\partial t \partial r}(a, c)\right|^{q}+(1-t) r\left|\frac{\partial^{2} f}{\partial t \partial r}(a, d)\right|^{q}\right. \\
& \left.\left.+t(1-r)\left|\frac{\partial^{2} f}{\partial t \partial r}(b, c)\right|^{q}+t r\left|\frac{\partial^{2} f}{\partial t \partial r}(b, d)\right|^{q}\right] d r d t\right)^{\frac{1}{q}} \\
& =\frac{a b(b-a) c d(d-c)}{4} \\
& \times\left(\left(b^{-2 p}{ }_{2} F_{1}\left(2 p, 1 ; 2 ; 1-\frac{a}{b}\right)\right) \times\left(d^{-2 p}{ }_{2} F_{1}\left(2 p, 1 ; 2 ; 1-\frac{c}{d}\right)\right)\right)^{\frac{1}{p}} \\
& \times\left(\left(\frac{1}{2(\alpha q+1)}\right)\left(\frac{1}{2(\beta q+1)}\right)\right. \\
& \left.\times\left[\left|\frac{\partial^{2} f}{\partial t \partial r}(a, c)\right|^{q}+\left|\frac{\partial^{2} f}{\partial t \partial r}(a, d)\right|^{q}+\left|\frac{\partial^{2} f}{\partial t \partial r}(b, c)\right|^{q}+\left|\frac{\partial^{2} f}{\partial t \partial r}(b, d)\right|^{q}\right]\right)^{\frac{1}{q}} .
\end{aligned}
$$

This completes the proof.

Theorem 3.5. Let $\mathrm{f}: \Delta \subset(0, \infty) \times(0, \infty) \rightarrow \mathbb{R}$ be partial differentiable function on $\Delta$ with $\mathrm{a}<\mathrm{b}$ and $\mathrm{c}<\mathrm{d}$. If $\frac{\partial^{2} \mathrm{f}}{\partial \mathrm{t} \partial \mathrm{r}} \in \mathscr{L}(\Delta)$ and $\left|\frac{\partial^{2} \mathrm{f}}{\partial \mathrm{t} \partial \mathrm{r}}\right|^{p}$ where $\frac{1}{\mathrm{p}}+\frac{1}{\mathrm{q}}=1$ is coordinated harmonic convex function, then

$$
\begin{aligned}
\Xi_{f}(\Delta ; \Gamma ; \alpha, \beta) \leqslant & \frac{a b(b-a) c d(d-c)}{4}\left(\frac{1}{(\alpha p+1)(\beta p+1)}\right)^{\frac{1}{p}} \\
& \times\left(\rho_{1}(\Delta ; \alpha, \beta)\left|\frac{\partial^{2} f}{\partial t \partial r}(a, c)\right|^{q}+\rho_{2}(\Delta ; \alpha, \beta)\left|\frac{\partial^{2} f}{\partial t \partial r}(a, d)\right|^{q}\right.
\end{aligned}
$$




$$
\left.+\rho_{3}(\Delta ; \alpha, \beta)\left|\frac{\partial^{2} f}{\partial t \partial r}(b, c)\right|^{q}+\rho_{1}(\Delta ; \alpha, \beta)\left|\frac{\partial^{2} f}{\partial t \partial r}(b, d)\right|^{q}\right)^{\frac{1}{q}}
$$

where

$$
\begin{aligned}
& \rho_{1}(\Delta ; \alpha, \beta)=\left\{\frac{\mathrm{b}^{-2 \mathrm{q}}}{2}{ }_{2} \mathrm{~F}_{1}\left(2 \mathrm{q}, 1 ; 3 ; 1-\frac{\mathrm{a}}{\mathrm{b}}\right)\right\} \times\left\{\frac{\mathrm{d}^{-2 \mathrm{q}}}{2}{ }_{2} \mathrm{~F}_{1}\left(2 \mathrm{q}, 1 ; 3 ; 1-\frac{\mathrm{c}}{\mathrm{d}}\right)\right\}, \\
& \rho_{2}(\Delta ; \alpha, \beta)=\left\{\frac{\mathrm{b}^{-2 \mathrm{q}}}{2}{ }_{2} \mathrm{~F}_{1}\left(2 \mathrm{q}, 1 ; 3 ; 1-\frac{\mathrm{a}}{\mathrm{b}}\right)\right\} \times\left\{\frac{\mathrm{d}^{-2 \mathrm{q}}}{2}{ }_{2} \mathrm{~F}_{1}\left(2 \mathrm{q}, 2 ; 3 ; 1-\frac{\mathrm{c}}{\mathrm{d}}\right)\right\}, \\
& \rho_{3}(\Delta ; \alpha, \beta)=\left\{\frac{\mathrm{b}^{-2 \mathrm{q}}}{2}{ }_{2} \mathrm{~F}_{1}\left(2 \mathrm{q}, 2 ; 3 ; 1-\frac{\mathrm{a}}{\mathrm{b}}\right)\right\} \times\left\{\frac{\mathrm{d}^{-2 q}}{2}{ }_{2} \mathrm{~F}_{1}\left(2 \mathrm{q}, 1 ; 3 ; 1-\frac{\mathrm{c}}{\mathrm{d}}\right)\right\},
\end{aligned}
$$

and

$$
\rho_{4}(\Delta ; \alpha, \beta)=\left\{\frac{b^{-2 q}}{2}{ }_{2} F_{1}\left(2 q, 2 ; 3 ; 1-\frac{a}{b}\right)\right\} \times\left\{\frac{d^{-2 q}}{2}{ }_{2} F_{1}\left(2 q, 2 ; 3 ; 1-\frac{c}{d}\right)\right\} .
$$

Proof. Using Lemma 2.1, property of the modulus, Hölder's inequality and the fact that $\left|\frac{\partial^{2} f}{\partial t \partial r}\right|^{p}$ is coordinated harmonic convex function, we have

$$
\begin{aligned}
\left|\Xi_{f}(\Delta ; \Gamma ; \alpha, \beta)\right|= & \frac{a b(b-a) c d(d-c)}{4} \\
& \times\left\{\iint_{0}^{1}\left(\frac{t^{\alpha}-(1-t)^{\alpha}}{A_{t}^{2}}\right)\left(\frac{r^{\beta}-(1-r)^{\beta}}{A_{r}^{2}}\right) \frac{\partial^{2} f}{\partial t \partial r}\left(\frac{a b}{A_{t}}, \frac{c d}{A_{r}}\right) d r d t\right\} \mid \\
\leqslant & \frac{a b(b-a) c d(d-c)}{4} \\
& \times\left(\int_{0}^{1} \int_{0}^{1}\left(\left|(1-t)^{\alpha}-t^{\alpha}\right|^{p}\right)\left(\left|(1-r)^{\beta}-r^{\beta}\right|^{p}\right) d r d t\right)^{\frac{1}{p}} \\
& \times\left(\int_{0}^{1} \int_{0}^{1} \frac{1}{A_{t}^{2 q} A_{r}^{2 q}} \times\left[(1-t)(1-r)\left|\frac{\partial^{2} f}{\partial t \partial r}(a, c)\right|^{q}+(1-t) r\left|\frac{\partial^{2} f}{\partial t \partial r}(a, d)\right|^{q}\right.\right. \\
& \left.\left.+t(1-r)\left|\frac{\partial^{2} f}{\partial t \partial r}(b, c)\right|^{q}+t r\left|\frac{\partial^{2} f}{\partial t \partial r}(b, d)\right|^{q}\right] d r d t\right)^{\frac{1}{q}} \\
\leqslant & \frac{a b(b-a) c d(d-c)}{4} \\
& \times\left(\int_{0}^{1} \int_{0}^{1}\left(|1-2 t|^{\alpha p}\right)\left(|1-2 r|^{\beta p}\right) d r d t\right)^{\frac{1}{p}} \\
& \times\left(\int_{0}^{1} \int_{0}^{1} \frac{1}{A_{t}^{2 q} A_{r}^{2 q}} \times\left[(1-t)(1-r)\left|\frac{\partial^{2} f}{\partial t \partial r}(a, c)\right|^{q}+(1-t) r\left|\frac{\partial^{2} f}{\partial t \partial r}(a, d)\right|^{q}\right.\right.
\end{aligned}
$$




$$
\begin{aligned}
& \left.\left.+t(1-r)\left|\frac{\partial^{2} f}{\partial t \partial r}(b, c)\right|^{q}+\operatorname{tr}\left|\frac{\partial^{2} f}{\partial t \partial r}(b, d)\right|^{q}\right] d r d t\right)^{\frac{1}{q}} \\
= & \frac{a b(b-a) c d(d-c)}{4}\left(\frac{1}{(\alpha p+1)(\beta p+1)}\right)^{\frac{1}{p}} \\
& \times\left(\rho_{1}(\Delta ; \alpha, \beta)\left|\frac{\partial^{2} f}{\partial t \partial r}(a, c)\right|^{q}+\rho_{2}(\Delta ; \alpha, \beta)\left|\frac{\partial^{2} f}{\partial t \partial r}(a, d)\right|^{q}\right. \\
& \left.+\rho_{3}(\Delta ; \alpha, \beta)\left|\frac{\partial^{2} f}{\partial t \partial r}(b, c)\right|^{q}+\rho_{1}(\Delta ; \alpha, \beta)\left|\frac{\partial^{2} f}{\partial t \partial r}(b, d)\right|^{q}\right)^{\frac{1}{q}},
\end{aligned}
$$

where

$$
\begin{aligned}
\rho_{1}(\Delta ; \alpha, \beta) & =\int_{0}^{1} \int_{0}^{1}(1-\mathrm{t})(1-\mathrm{r}) A_{\mathrm{t}}^{-2 \mathrm{q}} \mathrm{A}_{\mathrm{r}}^{-2 \mathrm{q}} \mathrm{drdt} \\
& =\left\{\frac{\mathrm{b}^{-2 \mathrm{q}}}{2}{ }_{2} \mathrm{~F}_{1}\left(2 \mathrm{q}, 1 ; 3 ; 1-\frac{\mathrm{a}}{\mathrm{b}}\right)\right\} \times\left\{\frac{\mathrm{d}^{-2 \mathrm{q}}}{2}{ }_{2} \mathrm{~F}_{1}\left(2 \mathrm{q}, 1 ; 3 ; 1-\frac{\mathrm{c}}{\mathrm{d}}\right)\right\}, \\
\rho_{2}(\Delta ; \alpha, \beta) & =\int_{0}^{1} \int_{0}^{1}(1-\mathrm{t}) \mathrm{r} \mathrm{A}_{\mathrm{t}}^{-2 \mathrm{q}} \mathrm{A}_{\mathrm{r}}^{-2 \mathrm{q}} \mathrm{drdt} \\
& =\left\{\frac{\mathrm{b}^{-2 \mathrm{q}}}{2}{ }_{2} \mathrm{~F}_{1}\left(2 \mathrm{q}, 1 ; 3 ; 1-\frac{\mathrm{a}}{\mathrm{b}}\right)\right\} \times\left\{\frac{\mathrm{d}^{-2 \mathrm{q}}}{2}{ }_{2} \mathrm{~F}_{1}\left(2 \mathrm{q}, 2 ; 3 ; 1-\frac{\mathrm{c}}{\mathrm{d}}\right)\right\}, \\
\rho_{3}(\Delta ; \alpha, \beta) & =\int_{0}^{1} \int_{0}^{1} \mathrm{t}(1-\mathrm{r}) \mathrm{A}_{\mathrm{t}}^{-2 \mathrm{q}} \mathrm{A}_{\mathrm{r}}^{-2 \mathrm{q}} \mathrm{drdt} \\
& =\left\{\frac{\mathrm{b}^{-2 \mathrm{q}}}{2}{ }_{2} \mathrm{~F}_{1}\left(2 \mathrm{q}, 2 ; 3 ; 1-\frac{\mathrm{a}}{\mathrm{b}}\right)\right\} \times\left\{\frac{\mathrm{d}^{-2 \mathrm{q}}}{2}{ }_{2} \mathrm{~F}_{1}\left(2 \mathrm{q}, 1 ; 3 ; 1-\frac{\mathrm{c}}{\mathrm{d}}\right)\right\},
\end{aligned}
$$

and

$$
\begin{aligned}
\rho_{4}(\Delta ; \alpha, \beta) & =\int_{0}^{1} \int_{0}^{1} \operatorname{tr} A_{t}^{-2 q} A_{r}^{-2 q} d r d t \\
& =\left\{\frac{b^{-2 q}}{2}{ }_{2} F_{1}\left(2 q, 2 ; 3 ; 1-\frac{a}{b}\right)\right\} \times\left\{\frac{d^{-2 q}}{2}{ }_{2} F_{1}\left(2 q, 2 ; 3 ; 1-\frac{c}{d}\right)\right\} .
\end{aligned}
$$

This completes the proof.

\section{Acknowledgment}

Authors are thankful to editor and anonymous referees for their constructive and valuable comments. Authors are pleased to acknowledge the "support of Distinguished Scientist Fellowship Program (DSFP), King Saud University," Riyadh, Saudi Arabia. 


\section{References}

[1] G. Cristescu, L. Lupşa, Non-connected convexities and applications, Applied Optimization, Kluwer Academic Publishers, Dordrecht, (2002). 1

[2] G. Cristescu, M. A. Noor, M. U. Awan, Bounds of the second degree cumulative frontier gaps of functions with generalized convexity, Carpathian J. Math., 31 (2015), 173-180.

[3] S. S. Dragomir, On the Hadamard's inequality for convex functions on the co-ordinates in a rectangle from the plane, Taiwanese J. Math., 5 (2001), 775-788.

[4] S. S. Dragomir, C. E. M. Pearce, Selected topics on Hermite-Hadamard inequalities and applications, RGMIA Monographs, Victoria University, (2000).

[5] D.-Y. Hwang, K.-L. Tseng, G.-S. Yang, Some Hadamard's inequalities for co-ordinated convex functions in a rectangle from the plane, Taiwanese J. Math., 11 (2007), 63-73.

[6] İ. İşcan, Hermite-Hadamard type inequalities for harmonically convex functions, Hacet. J. Math. Stat., 43 (2014), $935-942$. $1,1.1,1.2,1.6$

[7] İ. İşcan, S.-H. Wu, Hermite-Hadamard type inequalities for harmonically convex functions via fractional integrals, Appl. Math. Comput., 238 (2014), 237-244. 1

[8] S. K. Khattri, Three proofs of the inequality e $<\left(1+\frac{1}{n}\right)^{n+0.5}$, Amer. Math. Monthly, 117 (2010), 273-277. 1

[9] A. A. Kilbas, H. M. Srivastava, J. J. Trujillo, Theory and applications of fractional differential equations, North-Holland Mathematics Studies, Elsevier Science B.V., Amsterdam, (2006). 1.4

[10] M. A. Latif, S. Hussain, New inequalities of Ostrowski type for co-ordinated convex functions via fractional integrals, J. Fract. Calc. Appl., 2 (2012), 1-15. 1

[11] M. V. Mihai, New inequalities for co-ordinated convex functions via Riemann-Liouville fractional calculus, Tamkang J. Math., 45 (2014), 285-296. 1

[12] M. A. Noor, K. I. Noor, Harmonic variational inequalities, Appl. Math. Inf. Sci., 10 (2016), 1811-1814. 1

[13] M. A. Noor, K. I. Noor, M. U. Awan, Some characterizations of harmonically log-convex functions, Proc. Jangjeon Math. Soc., 17 (2014),51-61. 1

[14] M. A. Noor, K. I. Noor, M. U. Awan, Integral inequalities for coordinated harmonically convex functions, Complex Var. Elliptic Equ., 60 (2015), 776-786. 1, 1, 2

[15] M. A. Noor, K. I. Noor, M. U. Awan, S. Costache, Some integral inequalities for harmonically h-convex functions, Politehn. Univ. Bucharest Sci. Bull. Ser. A Appl. Math. Phys., 77 (2015), 5-16.

[16] M. A. Noor, K. I. Noor, M. U. Awan, J.-Y. Li, On Hermite-Hadamard inequalities for h-preinvex functions, Filomat, 28 (2014), 1463-1474.

[17] M. E. Ozdemir, Ç. Yıldız, A. O. Akdemir, On the co-ordinated convex functions, Appl. Math. Inf. Sci., 8 (2014), 1085-1091.

[18] J. Park, Hermite-Hadamard-like and Simpson-like type inequalities for harmonically convex functions, Int. J. Math. Anal., 8 (2014), 1321-1337.

[19] M. Z. Sarıkaya, On the Hermite-Hadamard-type inequalities for co-ordinated convex function via fractional integrals, Integral Transforms Spec. Funct., 25 (2014), 134-147. 1, 1.5

[20] M. Z. Sarıkaya, E. Set, M. E. Ozdemir, S. S. Dragomir, New some Hadamard's type inequalities for co-ordinated convex functions, Tamsui Oxf. J. Inf. Math. Sci., 28 (2012), 137-152.

[21] M. Z. Sarıkaya, E. Set, H. Yaldiz, N. Başak, Hermite-Hadamards inequalities for fractional integrals and related fractional inequalities, Math. Comput. Model., 57 (2013), 2403-2407. 1

[22] B.-Y. Xi, J. Hua, F. Qi, Hermite-Hadamard type inequalities for extended s-convex functions on the co-ordinates in a rectangle, J. Appl. Anal., 20 (2014), 29-39.

[23] T.-Y. Zhang, A.-P. Ji, F. Qi, Integral inequalities of Hermite-Hadamard type for harmonically quasi-convex functions, Proc. Jangjeon Math. Soc., 16 (2013), 399-407. 1 